SCIENCE CHINA

Physics, Mechanics \& Astronomy

\title{
Strengthening effects of various grain boundaries with nano-spacing as barriers of dislocation motion from molecular dynamics simulations
}

\author{
FuPing Yuan ${ }^{1,2^{*}}$ \\ ${ }^{1}$ State Key Laboratory of Nonlinear Mechanics, Institute of Mechanics, Chinese Academy of Science, Beijing 100190, China; \\ ${ }^{2}$ School of Engineering Science, University of Chinese Academy of Sciences, Beijing 100190, China
}

Received November 3, 2016; accepted December 20, 2016; published online January 9, 2017

\begin{abstract}
Strengthening in metals is traditionally achieved through the controlled creation of various grain boundaries (GBs), such as low-angle GBs, high-angle GBs, and twin boundaries (TBs). In the present study, a series of large-scale molecular dynamics simulations with spherical nanoindentation and carefully designed model were conducted to investigate and compare the strengthening effects of various GBs with nano-spacing as barriers of dislocation motion. Simulation results showed that high-angle twist GBs and TBs are similar barriers and low-angle twist GBs are less effective in obstructing dislocation motion. Corresponding atomistic mechanisms were also given. At a certain indentation depth, dislocation transmission and dislocation nucleation from the other side of boundaries were observed for low-angle twist GBs, whereas dislocations were completely blocked by high-angle twist GBs and TBs at the same indentation depth. The current findings should provide insights for comprehensive understanding of the strengthening effects of various GBs at nanoscale.
\end{abstract}

molecular dynamics, grain boundary strengthening, dislocations, nanoindentation, metals

PACS number(s): 46.15.-x, 46.55.+d, 61.72.Lk, 61.72.Mm, 71.15.Pd

Citation: F. P. Yuan, Strengthening effects of various grain boundaries with nano-spacing as barriers of dislocation motion from molecular dynamics simulations, Sci. China-Phys. Mech. Astron. 60, 034611 (2017), doi: 10.1007/s11433-016-0437-1

\section{Introduction}

Traditionally, strength of metals can be elevated by grain refinement through introducing more grain boundaries (GBs) or by storing more dislocations inside grain interiors $[1,2]$. Both strengthening mechanisms can be realized by severe plastic deformation (SPD) techniques, such as cold rolling (CR) [3], accumulative roll-bonding (ARB) [4], equal channel angular pressing (ECAP) [5,6], high-pressure torsion (HPT) [7], dynamic plastic deformation (DPD) [8], surface mechanical attrition treatment (SMAT) [9], and surface mechanical grinding treatment (SMGT) $[10,11]$.

\footnotetext{
*Corresponding author (email: fpyuan@1nm.imech.ac.cn)
}

Typically, the strength-structure relationship of metals can be described in terms of dislocation strengthening or boundary strengthening for different types of boundaries, as follows [12-14]: (1) strength elevation is inversely proportional to the square root of grain size for GBs with misorientation angles larger than $15^{\circ}$, which are typically called high-angle grain boundaries (HAGBs); (2) strength elevation is inversely proportional to the square root of the cell boundary spacing or is proportional to the square root of the dislocation density for GBs with misorientation angles less than $15^{\circ}$, which are typically called low-angle grain boundaries (LAGBs). As special GBs, twin boundaries (TBs) are also effective barriers because the strength elevation they provide is inversely proportional to the square root of TB spacing [15-19]. TBs can 
typically be introduced by electroplating deposition (growth twins) [15], plastic deformation (deformation twins) [20,21], or recrystallization of deformed structures upon annealing (annealing twins) [22]. Although the softening of metals with decreasing grain size/TB spacing can be observed when the grain size/TB spacing is below the critical grain size/TB spacing because of the deformation mechanisms of GB activities [15,17-19,23-28], HAGBs, LAGBs, and TBs can all obstruct dislocation motion for strengthening above the critical length scale during plastic deformation. Therefore, it is necessary to find out the abilities of various GBs to block dislocation motion and its underlying atomistic deformation mechanisms.

Previous research has shown molecular dynamics (MD) simulations to be successful in illustrating the plastic deformation mechanisms of various GBs under various deformation conditions, such as uniaxial tensile loading [23,26,28-31], simple shear loading [32,33], and nanoindentation [34-41]. MD simulations have advantages to take snapshots at any times and show the atomistic transient responses of microstructures. Moreover, transient stress and strain contours with atomic resolutions can also be obtained using MD simulations. Using $\mathrm{Ni}$ as a model material in a series of large-scale MD simulations, the focus of this research is to understand the strengthening effects of various GBs as barriers of dislocation motion and the underlying atomistic deformation mechanisms.

\section{Simulation techniques}

MD simulations were conducted using the large-scale atomic/molecular massively parallel simulator (LAMMPS) code, and force interactions between atoms are described by the Ni EAM potential developed by Mishin et al. [42]. Spherical indentation was applied, and the schematic of the simulation cell is shown in Figure 1, in which atoms are colored according to common neighbor analysis (CNA) values (this color coding is used throughout). Perfect fcc atoms are gray (but are not displayed in this figure), hcp atoms are red, and disordered atoms (GBs, dislocation core, free surface, etc.) are green. To explore the strengthening effects of various GBs, bilayers with (111) surface orientations ( $Z$ axis) were chosen. Boundaries in the simulation unit were created as follows: layer II was generated by rotating layer I about the $Z$ axis for different angles $\left(6^{\circ}\right.$ for twist LAGB and $90^{\circ}$ for twist HAGB) or by mirroring layer I about the $Z$ plane (TB). Because deformation in the $Z$ plane is isotropic for spherical indentation, layer II should be no difference from layer I for deformation response under spherical indentation; the Schmid factors for resolved shear stress acting on the possible slip systems in layer II should also be same as for layer I. The orientation effects should therefore be excluded, and the simulation unit shown in Figure 1(a) can be used to investigate solely the strengthening effects of various boundaries in obstructing dislocation motion. The detailed atomistic structures for the boundaries considered are shown in Figure 1(c). It should be noted that the twist LAGB is actually a dislocation wall consisting of periodic extended dislocations due to a small misorientation angle.

In Figure 1(a), the indentation was applied on the top free surface, and the bottom two atomic layers were set to be zero displacement in $Z$ direction for fixed boundaries during indentation. Periodic boundary conditions were imposed along the other two directions, i.e., $X$ and $Y$ directions. The size of the unit should be large enough to avoid self-interaction of dislocations according to the size of the indenter (in this case, $D=10 \mathrm{~nm}$ ) [40]. In this regard, dimensions of the simulation unit were approximately $60 \mathrm{~nm} \times 60 \mathrm{~nm} \times 44 \mathrm{~nm}$, containing $1.45 \times 10^{7}$ atoms. Layers I and II were repeated once along $Z$ direction to create a larger system for minimizing influence of the fixed bottom boundary (Figure 1(a)). The time step was set to be 2 fs and the velocity verlet algorithm was used during the simulations. By the conjugate gradient method, the as-created samples were subjected to energy minimization

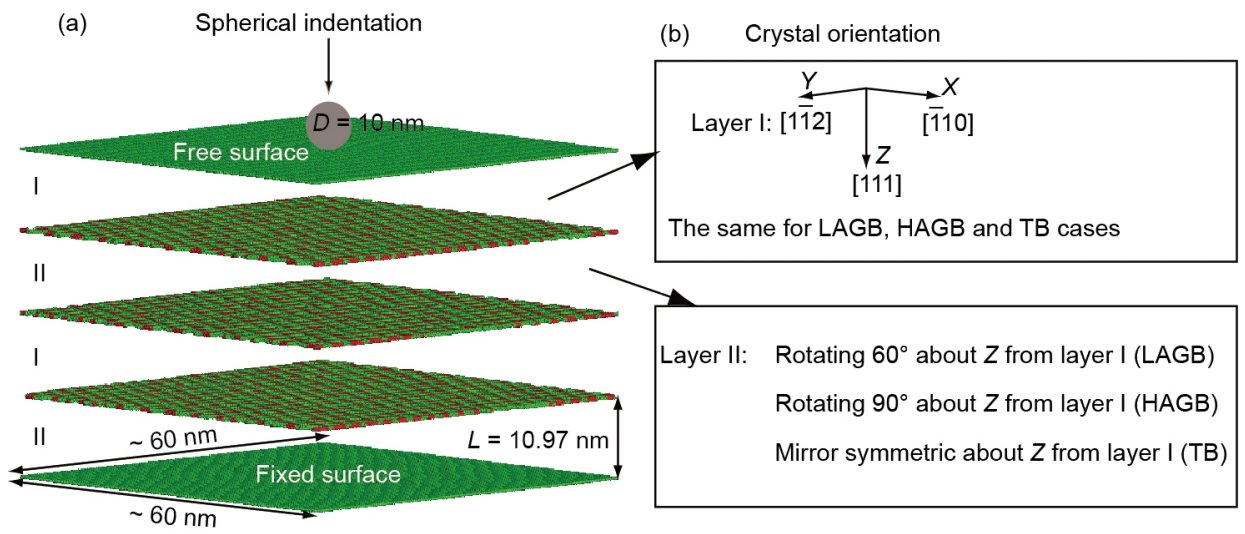

(c) Details of various GBs

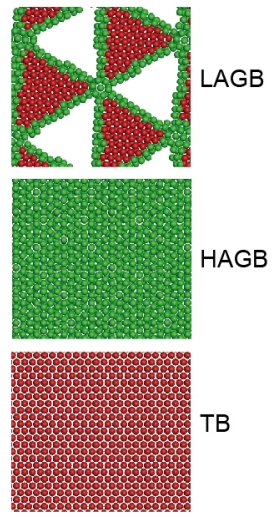

Figure 1 (Color online) (a) Schematic diagram of simulation unit for studying the strengthening effects of various GBs through spherical indentation; (b) crystal orientations for layer I and layer II in simulation unit for various GBs; (c) detailed microstructures of various GBs. 
before indentation. Then, using a Nose/Hoover isobaricisothermal ensemble (NPT), the samples were gradually heated up in a step-wise fashion to the desired temperature and finally relaxed under both the temperature of $1 \mathrm{~K}$ and zero pressure for $100 \mathrm{ps}$. During the indentation, the temperature of $1 \mathrm{~K}$ was maintained in the samples via an external Nose-Hoover thermostat to reduce the thermal effect, and a canonical ensemble (NVT) was used to relax the samples. Using displacement control, indentation was applied by moving a spherical indenter (initially positioned at the center point of the top free surface) at a constant downward velocity of $10 \mathrm{~m} / \mathrm{s}$ (this velocity should be much slower than the wave speed of $\mathrm{Ni}$, typically thousands of $\mathrm{m} / \mathrm{s}$ ). In the simulations, the indenter was virtual and was expressed with a repulsive potential

$F(r)= \begin{cases}-A(r-R)^{2}, & \text { for } r<R, \\ 0, & \text { for } r>R,\end{cases}$

where $A$ is the specified force constant for the force reaction between the virtual indenter and the atoms; $r$ is the distance from the virtual indenter center to the individual atoms; and $R(5 \mathrm{~nm})$ is the radius of the virtual indenter. In order to simulate the strong repulsive force between the indenter and the atoms below the indenter, the specified force constant should be large enough, in this case $A=1000 \mathrm{eV} / \AA^{2}$ as in previous research [41].

\section{Results and discussions}

Figure 2 displays the load curves as a function of indentation depth for various GBs. The elastic region and the onset of plasticity are revealed by a sharp drop in the indentation force and can be identified by the nucleation of the first dislocation beneath the spherical indenter, at the indentation depth of around $0.66 \mathrm{~nm}$. After the sharp drop at the onset of plastic deformation, the indentation force starts to increase again with increasing indentation depth, with a lower slope than the elastic region. At the indentation depth of around $1.2 \mathrm{~nm}$, the indentation force starts to increase with a much higher slope than in the early stage of plastic deformation, which indicates strong interactions between dislocations and the first GB. Previous research [37,43] has suggested that a planar GB/TB below or beside the indenter is an effective obstacle to the motion of dislocations and change the dominant dislocation patterns during the plastic deformation; thus it can provide greater resistance to the plastic flow than the single crystal. In summary, the load-depth response can be divided into three regions: (1) elastic region; (2) plastic region in which the dislocations generated by the indenter are far away from GBs and have no or weak interactions with GBs, so the indentation responses for various GBs should be similar and much like the single crystal in this region; and (3)

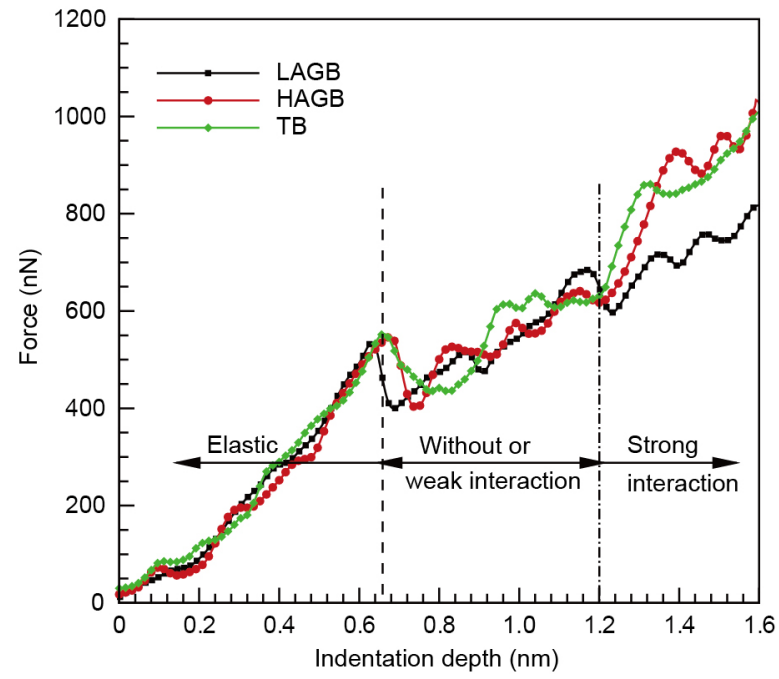

Figure 2 (Color online) Load-depth curves calculated from MD simulations for various GBs.

plastic region in which strong interactions are observed between dislocations and GBs, which means that different strengthening effects on obstructing dislocation motion by various GBs could be obtained in this region. As indicated in the third region of Figure 2, twist HAGB and TB were found to be similar in obstructing dislocation motion and twist LAGB was found to be a less effective barrier for dislocation motion.

Snapshots for atomistic microstructures were obtained at various indentation depths. Analysis of the deformed configurations is provided here in order to understand the strengthening mechanisms for various GBs under spherical indentation. Figure 3 shows the deformation snapshots for various GBs at the plastic region in which the deformation-induced dislocations have no or weak interactions with GBs. Figure 4 shows the deformation snapshots for various GBs at the plastic region in which strong interactions are observed between dislocations and GBs. In order to visualize the crystal defects more clearly, the perfect fcc atoms are not shown in Figures 3 and 4. As indicated in previous research [34], the dislocations might nucleate simultaneously from both underneath the indenter and the GBs or may even nucleate first from the GBs when the distance between the indenter and the GBs is small enough. This strengthening effect for GBs should be different from the case when the distance is large. In the present study, the distance between the indenter and the GBs was set to be large enough to generate dislocations first by indentation and then to investigate how various GBs obstruct these dislocations for strengthening.

At the onset of plasticity (Figure 3(a)), partial dislocations are produced first one after another at the area adjacent to the indentation site, and are then expanded into dislocation loops. These loops, which propagate along one of the $\{111\}$ slip planes, consist of two Shockley partial dislocations with 

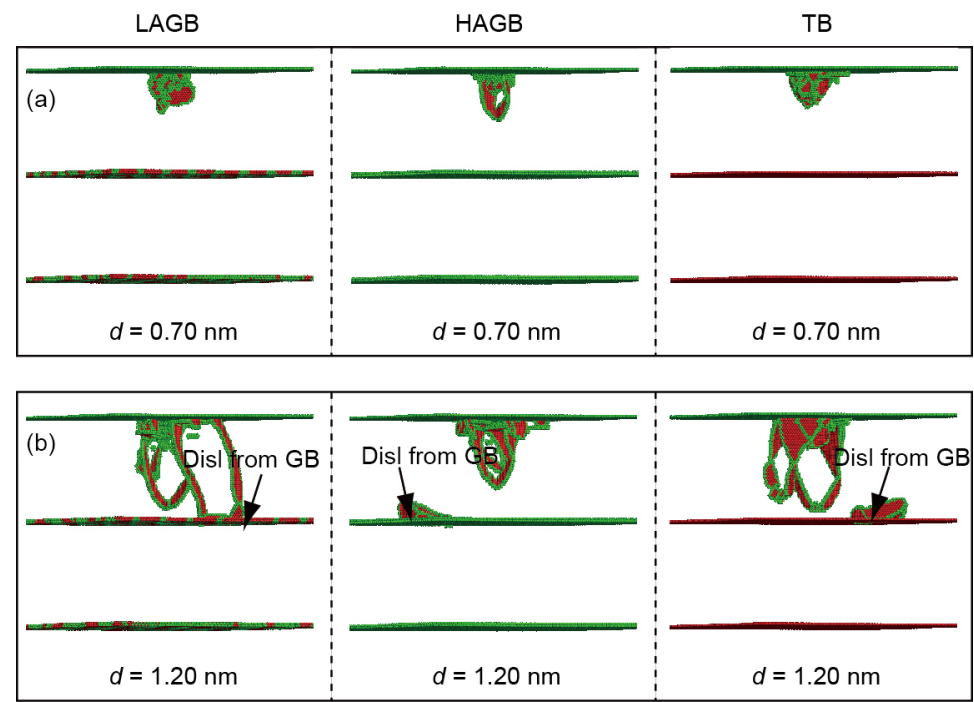

Figure 3 (Color online) Deformation snapshots showing the strengthening effects of various GBs as barriers of dislocation motion under spherical indentation at various depths. (a) $0.70 \mathrm{~nm}$; (b) $1.20 \mathrm{~nm}$.
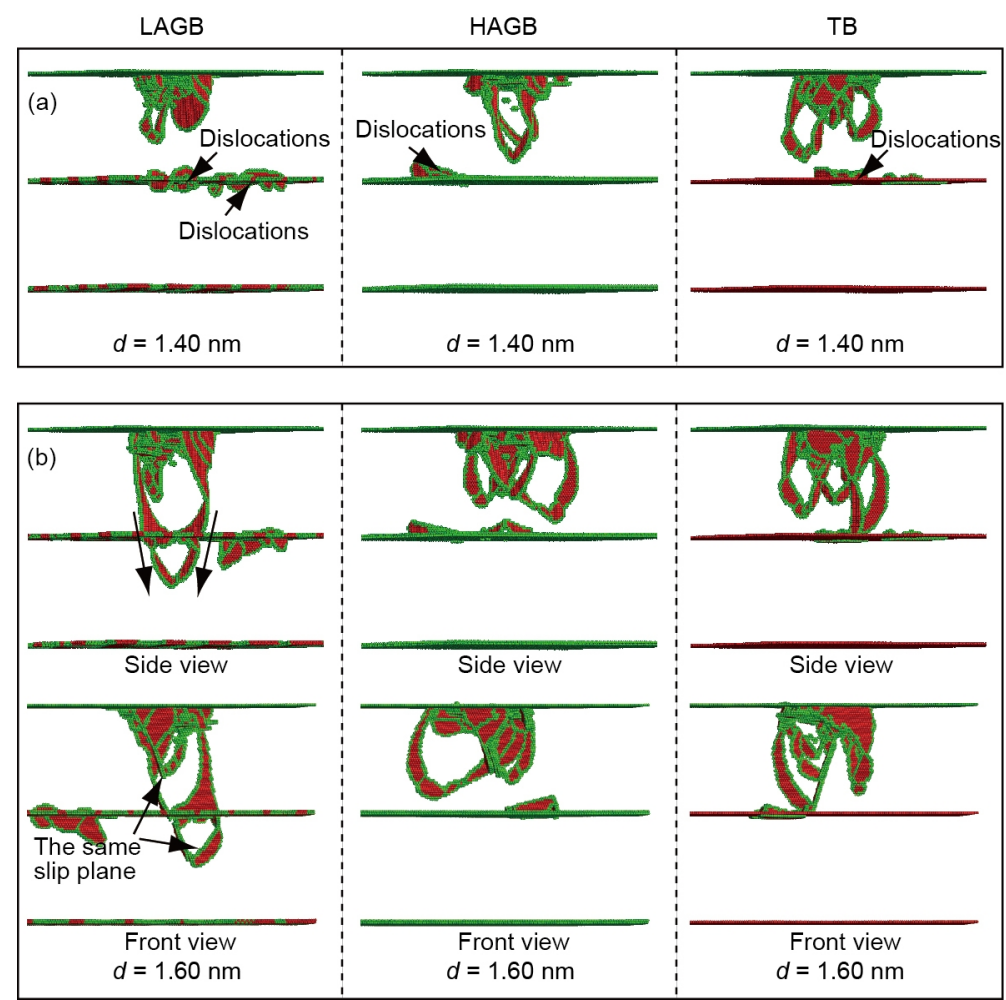

Figure 4 (Color online) Deformation snapshots showing the strengthenting effects of various GBs as barriers of dislocation motion under spherical indentation at various depths. (a) $1.40 \mathrm{~nm}$; (b) $1.60 \mathrm{~nm}$.

stacking faults between them. Detailed analysis of dislocations in the prismatic slip planes produced during nanoindentation, can be found elsewhere [43]. At an indentation depth of $1.2 \mathrm{~nm}$ (Figure 3(b)), the propagating dislocations are approaching the first GB and starting to take action on interactions between them and the GB, which results in the high slope in the load-depth curves at this point in Figure 2. At this point, a few partial dislocations also nucleate from the GB due to highly activated stresses on the GB at this stage.
With further increasing indentation depth $(1.4 \mathrm{~nm}$, Figure 4(a)), the dislocations interact with the twist LAGB and further propagation is delayed until a number of new partial dislocations appear on the other side of the twist LAGB (i.e., on the other grain across the twist LAGB). However, in the cases of twist HAGB and TB, the dislocations are absorbed and blocked completely by them without emitting new dislocations on the other side of the boundary at the same indentation depth. At even higher indentation depth 
(1.6 nm, both side and front views, Figure 4(b)), two dislocations are seen to transmit across the twist LAGB and into the adjacent grain. As suggested in previous research [37], dislocation transmission across the GB involves two steps: (1) the incoming dislocation is merged into the GB; and (2) the merged dislocation is dissociated into one lattice dislocation on the other side of the GB and another dislocation residing in the GB, which results in a step in the GB. This process starts with the reaction between the leading partial and the dislocations in the GB, followed by the reaction between the trailing partial and the dislocations in the GB. Although the detailed dislocation reactions are difficult to describe, the snapshots in Figure 5 clearly show the transmission process in which the dislocation above the GB is absorbed into the $\mathrm{GB}$ and a new dislocation at the same plane emits at the other side of the GB.

These results indicate that GBs can serve as barriers for dislocation motion and can resist plastic deformation, supporting the well-known Hall-Petch relationship for grain refinement $[1,2]$. Moreover, current results indicate that twist HAGBs and TBs can provide better resistance against penetration of incoming dislocations than twist LAGBs. Dislocation transmissions across GBs in metals have been studied previously by using in-situ TEM deformation techniques, and theories of dislocation transmissions based on geometric and stress rules have been proposed [44-46]. More recently, a concise review paper based on both experimental and atomistic simulation efforts have focused on the details of slip transmission mechanisms at GBs [47], and three criteria have been considered for predicting which slip system is favorable for slip transfer: (1) the slip system geometry criterion, the angle between the incoming and outgoing slip planes on two sides of the GB should be as small as possible; (2) the stress-based criterion, the resolved shear stress acting on the possible slip planes or slip systems in the adjacent grain should be large (under spherical indentation, the Schmid factors for resolved shear stress acting on the possible slip systems should be similar for various boundaries); and (3) criterion of residual GB dislocations, the residual Burgers vectors for dislocation reactions should be favorable [44-47]. Based on the first criterion, LAGBs with twist misorientation angles about the indentation direction are more prone to dislocation transmission under spherical nanoindentations, which is consistent with the current results. Furthermore, LAGBs can be considered to be an interfacial dislocation network, the incident dislocation interacts with the dislocation network, leaving residual dislocations in LAGBs. The residual Burgers vectors for dislocation reactions should be more favorable for dislocation transmission for LAGB case because the misorientation angle is smaller and the atom packing in LAGBs is less dense (Figure 1(c)) [47]. Therefore, the lower indentation force at the third region for LAGBs (Figure 2), could be attributed to stress release by the dislocation transmission through LAGBs and new dislocation nucleation at the adjacent grain/layer.

In order to understand the difference on the force reaction at the third region for various GBs, stress contours $\left(\sigma_{z z}\right)$ were provided. The stresses were calculated based on Virial theorem, and the atomic-level stress associated with each atom was obtained by decomposing the Virial stress onto individual atoms, in which the average volume was used for each atom [48]. A thin slice along the $Z$ direction with a thickness of 0.5 $\mathrm{nm}$ (the center of the slice is $5 \mathrm{~nm}$ away from the indentation surface) was considered, and the stress was averaged across the thickness. Figure 6 shows stress contours $\left(\sigma_{z z}\right)$ for various GBs at an indentation depth of $1.36 \mathrm{~nm}$, where strong interactions between dislocations and GBs take place. Most of the area beneath the indenter is under compression, indicated by the negative value of $\sigma_{z z}$. It is shown that the areas with compression stress for various GBs are very similar, while the high stress region (stress greater than $15 \mathrm{GPa}$, indicated in light blue and dark blue) is much larger in twist HAGB and TB cases than in twist LAGB case. The reaction force can be calculated by the integration of stress $\left(\sigma_{z z}\right)$ over the area, which means that the indentation forces in twist HAGB and TB cases should be larger than in twist LAGB case at this indentation depth based on Figure 6. This result is consistent
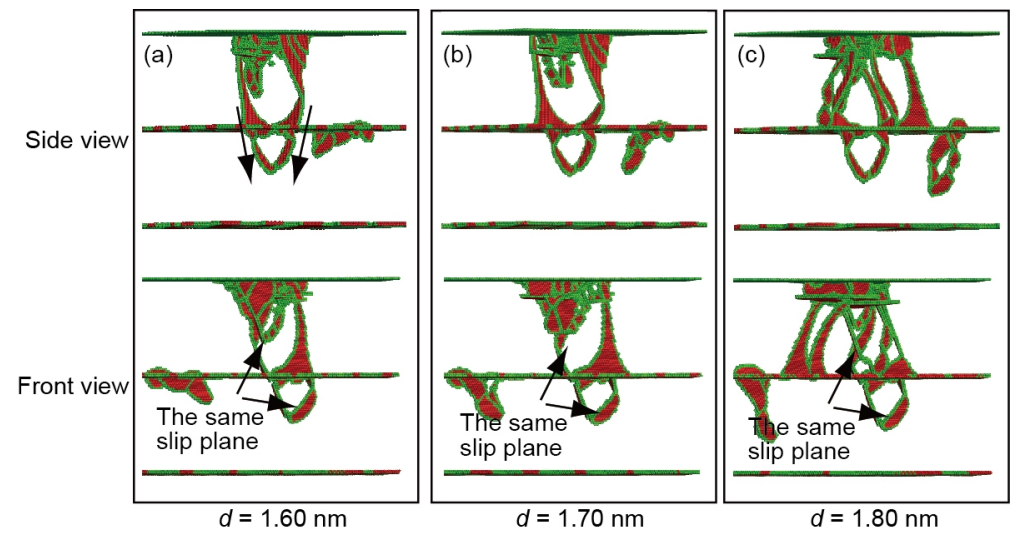

Figure 5 (Color online) Deformation snapshots at various depths showing the transmission process of dislocation across the LAGB. (a) 1.60 nm; (b) 1.70 nm; (c) $1.80 \mathrm{~nm}$. 

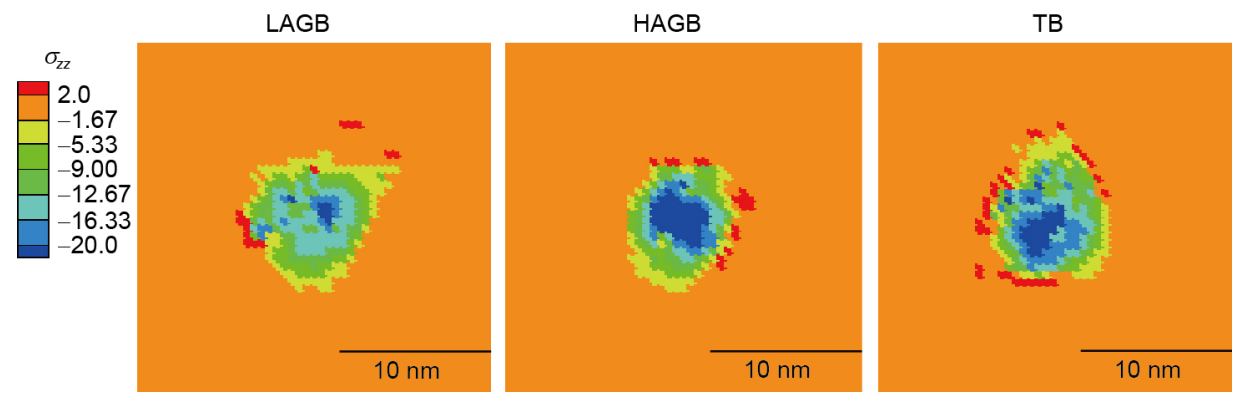

Figure 6 (Color online) Stress contours $\left(\sigma_{z z}\right)$ at an indentation depth of $1.36 \mathrm{~nm}$ for various GBs.

with the observations in Figure 2.

Current results indicated that discontinuous twist LAGBs are less effective in blocking dislocations than twist HAGBs and TBs. These results are consistent with previous suggestions $[16,49]$ that LAGBs with an array of aligned edge or screw dislocations may not be as effective at resisting transmission/penetration by moving dislocations as HAGBs. The transmission of a dislocation across randomly oriented HAGBs was not observed during the indentation process in other research $[34,35]$, which is similar to our oriented planar twist HAGB and TB cases at a certain indentation depth. However, dislocation transmission through twist LAGBs was observed in our simulations at the same indentation depth, which resulted in lower resistance to dislocation motion. The strength contribution from LAGBs is taken to be related to the density of dislocation stored in the LAGBs [12-14], therefore it may turn out that dislocation strengthening is not as effective as ordinary boundary strengthening under similar conditions. Moreover, strengthening of TBs was shown to be quantitatively similar to that of ordinary twist HAGBs in the present study. Four possible dislocation-TB reaction mechanisms have been indicated by previous research $[16,50]$. Among them, two mechanisms should provide strong strengthening and hardening through immobile dislocation locks, and these strengthening effects may be as strong as ordinary HAGBs as indicated in the present study. The softening effect can be observed due to the fact that twinning dislocations can glide along TBs for detwinning when the TB spacing is small enough [17]. The softening effect has also been observed for general GBs due to the GB sliding and the other GB activities when the grain size is smaller than the critical strain size [23]. It should be noted that there are two characteristic length scales in the present study, the indenter radius and the layer thickness. Under nanoindentation, the strengthening effect is likely to be different for different layer thickness, and those size effects for these two characteristic sizes will be further investigated in a future study.

\section{Summary}

The strengthening effects of various GBs as barriers of dislocation motion were investigated by spherical indentation through a series of large-scale MD simulations. High-angle twist GBs and TBs were found to be more effective than lowangle twist GBs in obstructing dislocation motion. Dislocation transmission and dislocation nucleation from the other side of boundaries were observed for low-angle twist GBs under a certain indentation depth, while dislocations were completely blocked by high-angle twist GBs and TBs at the same indentation depth. The current findings should enhance our understanding of strengthening metals by various GBs.

This work was supported by the National Natural Science Foundation of China (Grant Nos. 11472286, and 11672313), the National Key Basic Research Program of China (Grants Nos. 2012CB932203, and 2012CB937500), and the Strategic Priority Research Program of the Chinese Academy of Sciences (Grant No. XDB22040503).

1 M. A. Meyers, A. Mishra, and D. J. Benson, Prog. Mater. Sci. 51, 427 (2006)

2 M. Dao, L. Lu, R. Asaro, J. Dehosson, and E. Ma, Acta Mater. 55, 4041 (2007).

3 D. A. Hughes, and N. Hansen, Acta Mater. 48, 2985 (2000).

4 N. Tsuji, Y. Ito, Y. Saito, and Y. Minamino, Scr. Mater. 47, 893 (2002).

5 R. Z. Valiev, and T. G. Langdon, Prog. Mater. Sci. 51, 881 (2006).

6 I. J. Beyerlein, and L. S. Tóth, Prog. Mater. Sci. 54, 427 (2009).

7 A. Zhilyaev, and T. Langdon, Prog. Mater. Sci. 53, 893 (2008).

8 Y. S. Li, N. R. Tao, and K. Lu, Acta Mater. 56, 230 (2008).

9 K. Wang, N. R. Tao, G. Liu, J. Lu, and K. Lu, Acta Mater. 54, 5281 (2006).

10 T. H. Fang, W. L. Li, N. R. Tao, and K. Lu, Science 331, 1587 (2011).

11 X. C. Liu, H. W. Zhang, and K. Lu, Science 342, 337 (2013).

12 N. Kamikawa, X. Huang, N. Tsuji, and N. Hansen, Acta Mater. 57, 4198 (2009).

13 Z. P. Luo, H. W. Zhang, N. Hansen, and K. Lu, Acta Mater. 60, 1322 (2012).

14 K. S. Kumar, H. Van Swygenhoven, and S. Suresh, Acta Mater. 51, 5743 (2003).

15 L. Lu, X. Chen, X. Huang, and K. Lu, Science 323, 607 (2009).

16 K. Lu, L. Lu, and S. Suresh, Science 324, 349 (2009).

17 X. Li, Y. Wei, L. Lu, K. Lu, and H. Gao, Nature 464, 877 (2010).

18 F. Yuan, and X. Wu, Phys. Rev. B 86, 134108 (2012).

19 Y. T. Zhu, X. Z. Liao, and X. L. Wu, Prog. Mater. Sci. 57, 1 (2012).

20 M. Chen, E. Ma, K. J. Hemker, H. Sheng, Y. Wang, and X. Cheng, Science 300, 1275 (2003).

21 I. J. Beyerlein, L. Capolungo, P. E. Marshall, R. J. McCabe, and C. N. Tomé, Philos. Mag. 90, 2161 (2010).

22 D. Field, L. Bradford, M. Nowell, and T. Lillo, Acta Mater. 55, 4233 (2007).

23 J. Schiøtz, and K. W. Jacobsen, Science 301, 1357 (2003). 
24 K. Kadau, P. S. Lomdahl, B. L. Holian, T. C. Germann, D. Kadau, P. Entel, D. E. Wolf, M. Kreth, and F. Westerhoff, Metall. Mat. Trans. A 35, 2719 (2004).

25 T. G. Nieh, and J. G. Wang, Intermetallics 13, 377 (2005).

26 J. B. Jeon, B. J. Lee, and Y. W. Chang, Scr. Mater. 64, 494 (2011).

27 N. Q. Vo, R. S. Averback, P. Bellon, and A. Caro, Phys. Rev. B 78, 241402 (2008).

28 Z. B. Qi, P. Sun, F. P. Zhu, Z. C. Wang, D. L. Peng, and C. H. Wu, Surf. Coat. Tech. 205, 3692 (2011).

29 M. A. Tschopp, and D. L. McDowell, Scr. Mater. 58, 299 (2008).

30 D. Farkas, and L. Patrick, Philos. Mag. 89, 3435 (2009).

31 F. P. Yuan, Sci. China-Phys. Mech. Astron. 55, 1657 (2012).

32 J. W. Cahn, and J. E. Taylor, Acta Mater. 52, 4887 (2004).

33 J. W. Cahn, Y. Mishin, and A. Suzuki, Acta Mater. 54, 4953 (2006).

34 D. Feichtinger, P. M. Derlet, and H. Van Swygenhoven, Phys. Rev. B 67, 024113 (2003).

35 A. Hasnaoui, P. M. Derlet, and H. Van Swygenhoven, Acta Mater. 52, 2251 (2004).

36 S. Qu, and H. Zhou, Nanotechnology 21, 335704 (2010).

37 H. Jang, and D. Farkas, Mater. Lett. 61, 868 (2007).
38 Y. Kulkarni, R. Asaro, and D. Farkas, Scr. Mater. 60, 532 (2009).

39 F. Sansoz, and K. D. Stevenson, Phys. Rev. B 83, 224101 (2011).

40 I. H. Chen, C. I. Hsiao, R. K. Behera, and W. D. Hsu, J. Appl. Phys. 114, 214305 (2013).

41 X. Liu, F. Yuan, and Y. Wei, Appl. Surf. Sci. 279, 159 (2013).

42 Y. Mishin, D. Farkas, M. J. Mehl, and D. A. Papaconstantopoulos, Phys. Rev. B 59, 3393 (1999).

43 K. J. Kim, J. H. Yoon, M. H. Cho, and H. Jang, Mater. Lett. 60, 3367 (2006).

44 T. C. Lee, I. M. Robertson, and H. K. Birnbaum, Philos. Mag. A 62, 131 (1990).

45 Z. Shen, R. H. Wagoner, and W. A. T. Clark, Scr. Metall. 20, 921 (1986).

46 J. D. Livingston, and B. Chalmers, Acta Metall. 5, 322 (1957).

47 D. E. Spearot, and M. D. Sangid, Curr. Opin. Solid State Mater. Sci. 18, 188 (2014).

48 A. Cao, Y. Wei, and E. Ma, Phys. Rev. B 77, 195429 (2008).

49 W. J. Babyak, and F. N. Rhines, Trans. Met. Soc. AIME 218, 21 (1960).

50 L. Lu, Z. S. You, and K. Lu, Scr. Mater. 66, 837 (2012). 